\title{
On the Deformation Retract of Kerr Spacetime and Its Folding
}

\author{
H. Rafat \\ Department of Mathematics, Faculty of Science, Tanta University, Tanta 31111, Egypt \\ Correspondence should be addressed to H. Rafat; hishamrafat2000@yahoo.com
}

Received 26 May 2014; Revised 8 September 2014; Accepted 9 September 2014; Published 10 November 2014

Academic Editor: Shao-Ming Fei

Copyright (C) $2014 \mathrm{H}$. Rafat. This is an open access article distributed under the Creative Commons Attribution License, which permits unrestricted use, distribution, and reproduction in any medium, provided the original work is properly cited.

The deformation retract of the Kerr spacetime is introduced using Lagrangian equations. The equatorial geodesics of the Kerr space have been discussed. The retraction of this space into itself and into geodesics has been presented. The deformation retract of this space into itself and after the isometric folding has been discussed. Theorems concerning these relations have been deduced.

\section{Introduction}

The real revolution in mathematical physics in the second half of twentieth century (and in pure mathematics itself) was algebraic topology and algebraic geometry [1]. In the nineteenth century, mathematical physics was essentially the classical theory of ordinary and partial differential equations. The variational calculus, as a basic tool for physicists in theoretical mechanics, was seen with great reservation by mathematicians until Hilbert set up its rigorous foundation by pushing forward functional analysis. This marked the transition into the first half of twentieth century, where, under the influence of quantum mechanics and relativity, mathematical physics turned mainly into functional analysis, complemented by the theory of Lie groups and by tensor analysis. All branches of theoretical physics still can expect the strongest impacts of use of the unprecedented wealth of results of algebraic topology and algebraic geometry of the second half of the twentieth century [1].

Today, the concepts and methods of topology and geometry have become an indispensable part of theoretical physics. They have led to a deeper understanding of many crucial aspects in condensed matter physics, cosmology, gravity, and particle physics. Moreover, several intriguing connections between only apparently disconnected phenomena have been revealed based on these mathematical tools $[2,3]$.

Topology enters general relativity through the fundamental assumption that spacetime exists and is organized as a manifold. This means that spacetime has a well-defined dimension, but it also carries with it the inherent possibility of modified patterns of global connectivity, such as distinguishing a sphere from a plane or a torus from a surface of higher genus. Such modifications can be present in the spatial topology without affecting the time direction, but they can also have a genuine spacetime character in which case the spatial topology changes with time [4]. The topology change in classical general relativity has been discussed in [5]. See [6] for some applications of differential topology in general relativity.

In general relativity, boundaries that are $S^{1}$ bundles over some compact manifolds arise in gravitational thermodynamics [7]. The trivial bundle $\sum=S^{1} \times S^{2}$ is a classic example. Manifolds with complete Ricci-flat metrics admitting such boundaries are known; they are the Euclideanised Schwarzschild metric and the flat metric with periodic identification. York [8] shows that there are in general two or no Schwarzschild solutions depending on whether the squashing (the ratio of the radius of the $S^{1}$-fibre to that of the $S^{2}$-base) is below or above a critical value. York's results in 4-dimension extend readily to higher dimensions.

The simplest example of nontrivial bundles arises in quantum cosmology in which the boundary is a compact $S^{3}$, that is, a nontrivial $S^{1}$ bundle over $S^{2}$. In the case of zero cosmological constant, regular 4 metrics admitting such an $S^{3}$ boundary are the Taub-Nut [9] and Taub-Bolt [10] metrics having zero and two-dimensional (regular) fixed point sets of the $U(1)$ action, respectively $[7,11-13]$. 
The Kerr metric describes the geometry of empty spacetime around a rotating uncharged axially symmetric black hole. The Kerr metric corresponds to the line element

$$
\begin{aligned}
& d s^{2}=-\left(1-\frac{2 M r}{\rho^{2}}\right) d t^{2}-\frac{4 M r a \sin ^{2} \theta}{\rho^{2}} d \phi d t \\
&+\frac{\rho^{2}}{\Delta} d r^{2}+\rho^{2} d^{2} \theta \\
&+\left(r^{2}+a^{2}+\frac{2 M r a^{2} \sin ^{2} \theta}{\rho^{2}}\right) \sin ^{2} \theta d^{2} \phi, \\
& a=\frac{J}{M}, \quad \rho^{2}=r^{2}+a^{2} \cos ^{2} \theta, \\
& \Delta=r^{2}-2 M r+a^{2} .
\end{aligned}
$$

The parameter $a$, termed the Kerr parameter, has units of length in geometrized units. The parameter $J$ will be interpreted as angular momentum and the parameter $M$ will be interpreted as the mass for the black hole. The Kerr metric is a vacuum solution of the Einstein equations, being valid in the absence of matter. If the black hole is not rotating $(a=J / M=$ 0 ), the Kerr line element reduces to the Schwarzschild line element. The Kerr metric becomes asymptotically flat for $r \gg M$ and $r \gg a$. Unlike the Schwarzschild metric, the Kerr metric has only axial symmetry.

\section{Deformation Retract}

2.1. Deformation Retract Definitions. The theory of deformation retract is very interesting topic in Euclidean and non-Euclidean spaces. It has been investigated from different points of view in many branches of topology and differential geometry. A retraction is a continuous mapping from the entire space into a subspace which preserves the position of all points in that subspace [14].

(i) Let $M$ and $N$ be two smooth manifolds of dimensions $m$ and $n$, respectively. A map $f: M \rightarrow N$ is said to be an isometric folding of $M$ into $N$ if and only if, for every piecewise geodesic path $\gamma: J \rightarrow M$, the induced path $f \circ \gamma: J \rightarrow N$ is a piecewise geodesic and of the same length as $\gamma$ [15]. If $f$ does not preserve the lengths, it is called topological folding. Many types of foldings are discussed in [16-21]. Some applications are discussed in [22, 23].

(ii) A subset $A$ of a topological space $X$ is called a retraction of $X$ if there exists a continuous map $r: X \rightarrow A$ such that [24]
(a) $X$ is open;
(b) $r(a)=a, \forall a \in A$.

(iii) A subset $A$ of a topological space $X$ is said to be a deformation retract if there exist a retraction $r: X \rightarrow A$ and a homotopy $H: X \times[0,1] \rightarrow X$ such that [24]

$$
\begin{gathered}
H(x, 0)=x, \quad \forall x \in X, \\
H(x, 1)=r(x), \quad \forall a \in X, \\
H(a, t)=a, \quad \forall a \in A, t \in[0,1] .
\end{gathered}
$$

The deformation retract is a particular case of homotopy equivalence and two spaces are homotopy equivalent if and only if they are both deformation retracts of a single larger space.

Deformation retracts of Stein spaces have been studied in [25]. The deformation retract of the $4 \mathrm{D}$ Schwarzschild metric has been discussed in [26] where it was found that the retraction of the Schwarzschild space is spacetime geodesic. The 5-dimensional case has been discussed in [27].

\section{Equatorial Geodesics}

We will be interested in the equatorial geodesics, that is, geodesics with $\theta=\pi / 2$. It is easy to show that such geodesics exist for the case of Kerr metric where $\theta=\pi / 2$ satisfies the $\theta$-component of the Euler Lagrange equations for the Lagrangian associated with the Kerr metric (1). Consider

$$
\begin{aligned}
L= & -\frac{1}{2}\left(1-\frac{2 M r}{\rho^{2}}\right) \dot{t}^{2}-\frac{2 M r a \sin ^{2} \theta}{\rho^{2}} \dot{\phi} \dot{t}+\frac{\rho^{2}}{2 \Delta} \dot{r}^{2} \\
& +\frac{1}{2} \rho^{2} \dot{\theta}^{2}+\frac{1}{2}\left(r^{2}+a^{2}+\frac{2 M r a^{2} \sin ^{2} \theta}{\rho^{2}}\right) \sin ^{2} \theta \dot{\phi}^{2} .
\end{aligned}
$$

The $\theta$-component of the Euler Lagrange equations gives

$$
\begin{aligned}
\frac{d}{d \lambda}\left(\rho^{2} \dot{\theta}\right)+\frac{2 M r a^{2} \sin \theta \cos \theta}{\rho^{4}} \dot{t}^{2}-\frac{a^{2} \sin \theta \cos \theta}{\Delta} \dot{r}^{2} \\
\quad-(\sin \theta \cos \theta) \dot{\theta}^{2}-\frac{4 M r a \sin \theta \cos \theta\left(\rho^{2}+a^{2} \sin \theta\right)}{\rho^{4}} \dot{t} \dot{\phi} \\
+\left(r^{2}+a^{2}\right) \sin \theta \cos \theta \dot{\phi}^{2}+\frac{1}{\rho^{4}} 4 M r a^{2} \sin \theta \cos \theta \\
\quad \times\left(a^{2} \sin ^{4} \theta+2\left(r^{2}+a^{2} \cos \theta\right) \sin \theta\right)=0 .
\end{aligned}
$$

Comparing the Kerr line element,

$$
\begin{aligned}
d s^{2}= & -\left(1-\frac{2 M}{r}\right) d t^{2}-\frac{4 M a}{r} d \phi d t \\
& +\frac{r^{2}}{\Delta} d r^{2}+\left(r^{2}+a^{2}+\frac{2 M a^{2}}{r}\right) d^{2} \phi .
\end{aligned}
$$

And the four-dimensional flat metric

$$
d s^{2}=d x_{1}^{2}+d x_{2}^{2}+d x_{3}^{2}+d x_{4}^{2} .
$$


The coordinates of the four-dimensional Kerr space (6) can be written as

$$
\begin{aligned}
& x_{1}=\left(r^{2}-4 M r+\left(2 M r-2 M^{2}\right) \ln \left(-r^{2}-a^{2}+2 M r\right)\right. \\
&+\frac{\tan ^{-1}\left((M-r) / \sqrt{a^{2}-M^{2}}\right)}{\sqrt{a^{2}-M^{2}}} \\
& \times\left(8 M^{3}-6 a^{2} M+2 a^{2} r-4 M^{2} r\right)+\left(a^{2}-2 M^{2}\right) \\
&\left.\times \ln \left(\frac{2 M r-r^{2}-a^{2}}{(M-a)(M+a)}\right)+C_{1}\right)^{1 / 2}, \\
& x_{2}= \pm \sqrt{\frac{\pi^{2}}{4} r^{2}+\pi^{2} 8 a^{2}+C_{2},} \\
& x_{3}= \pm \sqrt{\left(\left(r^{2}+a^{2}\right)+\frac{2 M a^{2}}{r}\right) \phi^{2}+C_{3}}, \\
& x_{4}= \pm \sqrt{\left(1-\frac{2 M}{r}\right) t^{2}+C_{4} .}
\end{aligned}
$$

In general relativity, the geodesic equation is equivalent to the Euler Lagrange equations

$$
\frac{d}{d \lambda}\left(\frac{\partial L}{\partial \dot{x}^{\alpha}}\right)-\frac{\partial L}{\partial x^{\alpha}}=0, \quad i=1,2,3,4
$$

associated to the Lagrangian

$$
L\left(x^{M}, \dot{x}^{M}\right)=\frac{1}{2} g_{M \nu} \dot{x}^{M} \dot{x}^{\nu}
$$

To find a geodesic which is a subset of the Kerr space, the Lagrangian could be written as

$$
\begin{aligned}
L= & -\frac{1}{2}\left(1-\frac{2 M}{r}\right) \dot{t}^{2}-\frac{2 M a}{r} \dot{\phi} \dot{t}+\frac{r^{2}}{2 \Delta} \dot{r}^{2} \\
& +\frac{1}{2}\left(r^{2}+a^{2}+\frac{2 M a^{2}}{r}\right) \dot{\phi}^{2} .
\end{aligned}
$$

There is no explicit dependence on $t$ or $\phi$; thus $\partial L / \partial \dot{t}=0$ and $\partial L / \partial \dot{\phi}=0$ are constants of motion; that is,

$$
\begin{gathered}
-\left(1-\frac{2 M}{r}\right) \dot{t}-2 \frac{M a}{r} \dot{\phi}=K_{1}, \\
-\frac{2 M a}{r} \dot{t}+\left(r^{2}+a^{2}+2 \frac{M a^{2}}{r}\right) \dot{\phi}=K_{2} .
\end{gathered}
$$

So we have the following set of equations:

$$
\begin{gathered}
\frac{d}{d \lambda}\left(\frac{r^{2} \dot{r}}{r^{2}+a^{2}-2 M r}\right)-\left(-\frac{M}{r^{2}} \dot{t}^{2}+\frac{r \dot{r}^{2}}{r^{2}+a^{2}-2 M r}\right. \\
\left.+\left(r-\frac{M a^{2}}{r^{2}}\right) \dot{\phi}^{2}\right)=0, \\
\frac{d}{d \lambda}\left[-\left(1-\frac{2 M}{r}\right) \dot{t}-2 \frac{M a}{r} \dot{\phi}\right]=0, \\
\frac{d}{d \lambda}\left[-\frac{2 M a}{r} \dot{t}+\left(r^{2}+a^{2}+2 \frac{M a^{2}}{r}\right) \dot{\phi}\right]=0 .
\end{gathered}
$$

If the constant $a$ is zero, we have

$$
\begin{aligned}
x_{1}=( & r^{2}-4 M r+\left(2 M r-2 M^{2}\right) \\
& \times \ln \left(-r^{2}+2 M r\right)+\frac{\tan ^{-1}((M-r) / M)}{M} \\
& \left.\times\left(8 M^{3}-4 M^{2} r\right)+\left(2 M^{2}\right) \ln \left(\frac{2 M r-r^{2}}{M^{2}}\right)+C_{1}\right)^{1 / 2},
\end{aligned}
$$

$x_{2}= \pm \sqrt{\frac{\pi^{2}}{4} r^{2}+C_{2}}$,

$x_{3}= \pm \sqrt{r^{2} \phi^{2}+C_{3}}$

$x_{4}= \pm \sqrt{\left(1-\frac{2 M}{r}\right) t^{2}+C_{4}}$.

Since $x_{1}^{2}+x_{2}^{2}+x_{3}^{2}-x_{4}^{2}>0$ which is the great circle $S_{1}$ in the Kerr space $K$, this geodesic is a retraction in Kerr space; $d s^{2}>0$. This is a retraction.

For $a=0$, (12) becomes

$$
\begin{gathered}
-\left(1-\frac{2 M}{r}\right) \dot{t}=K_{1}, \\
r^{2} \dot{\phi}=K_{2} .
\end{gathered}
$$

If $K_{1}=0$, then $t=$ const. If the constant is zero, then

$$
\begin{aligned}
& x_{1}=\frac{1}{2} r^{2}+M^{2}-M r+M \ln (r-M) r-M^{2} \ln (r-M), \\
& x_{2}= \pm \sqrt{\frac{\pi^{2}}{4} r^{2}+C_{2}} \\
& x_{3}= \pm \sqrt{r^{2} \phi^{2}+C_{3}} \\
& x_{4}= \pm \sqrt{C_{4}} .
\end{aligned}
$$

Since $x_{1}^{2}+x_{2}^{2}+x_{3}^{2}-x_{4}^{2}>0$ which is the great circle $S_{2}$ in the Kerr space $K$, this geodesic is a retraction in Kerr space; $d s^{2}>0$. 
If $K_{2}=0$, then $\phi=$ const. If the constant is zero, then

$$
\begin{aligned}
& x_{1}=\frac{1}{2} r^{2}+M^{2}-M r+M \ln (r-M) r-M^{2} \ln (r-M), \\
& x_{2}= \pm \sqrt{\frac{\pi^{2}}{4} r^{2}+C_{2}}, \\
& x_{3}= \pm \sqrt{C_{3}}, \\
& x_{4}= \pm \sqrt{\left(1-\frac{2 M}{r}\right) t^{2}+C_{4}} .
\end{aligned}
$$

Since $x_{1}^{2}+x_{2}^{2}+x_{3}^{2}-x_{4}^{2}>0$ which is the great circle $S_{3}$ in the Kerr space $K$, this geodesic is a retraction in Kerr space; $d s^{2}>0$.

From the above discussion, the following theorem has been proved.

Theorem 1. The retraction of the Kerr space is a geodesic in the Kerr space.

\section{Deformation Retract of Kerr Space}

The deformation retract of the Kerr space $K$ is defined as

$$
\phi: K \times I \longrightarrow K
$$

where $I$ is the closed interval $[0,1]$. The retraction of the Kerr space $K$ is defined as

$$
R: K \longrightarrow S_{1}, S_{2}, S_{3}
$$

Then, the deformation retract of the Kerr space $K$ into a geodesic $S_{1} \subset K$ is defined by

$$
\begin{aligned}
& \phi(M, c) \\
& =\cos \frac{\pi c}{2}\left\{\left(r^{2}-4 M r+\left(2 M r-2 M^{2}\right)\right.\right. \\
& \quad \times \ln \left(-r^{2}-a^{2}+2 M r\right) \\
& +\frac{\tan ^{-1}\left((M-r) / \sqrt{a^{2}-M^{2}}\right)}{\sqrt{a^{2}-M^{2}}} \\
& \quad \times\left(8 M^{3}-6 a^{2} M+2 a^{2} r-4 M^{2} r\right) \\
& +\left(a^{2}-2 M^{2}\right)
\end{aligned}
$$

$$
\begin{aligned}
& \left.\times \ln \left(\frac{2 M r-r^{2}-a^{2}}{(M-a)(M+a)}\right)+C_{1}\right)^{1 / 2}, \\
& \pm \sqrt{\frac{\pi^{2}}{4} r^{2}+\pi^{2} 8 a^{2}+C_{2}} \\
& \pm \sqrt{\left(\left(r^{2}+a^{2}\right)+\frac{2 M a^{2}}{r}\right) \phi^{2}+C_{3}} \text {, } \\
& \left. \pm \sqrt{\left(1-\frac{2 M}{r}\right) t^{2}+C_{4}}\right\} \\
& +\sin \frac{\pi c}{2}\left\{\frac{1}{2} r^{2}+M^{2}-M r+M \ln (r-M) r\right. \\
& -M^{2} \ln (r-M), \pm \sqrt{\frac{\pi^{2}}{4} r^{2}+C_{2}}, \\
& \left. \pm \sqrt{r^{2} \phi^{2}+C_{3}}, \pm \sqrt{\left(1-\frac{2 M}{r}\right) t^{2}+C_{4}}\right\} \text {, }
\end{aligned}
$$

where

$$
\begin{aligned}
& \phi(M, 0)=\left\{\left(r^{2}-4 M r+\left(2 M r-2 M^{2}\right)\right.\right. \\
& \times \ln \left(-r^{2}-a^{2}+2 M r\right) \\
& +\frac{\tan ^{-1}\left((M-r) /\left(\sqrt{a^{2}-M^{2}}\right)\right)}{\sqrt{a^{2}-M^{2}}} \\
& \times\left(8 M^{3}-6 a^{2} M+2 a^{2} r-4 M^{2} r\right) \\
& +\left(a^{2}-2 M^{2}\right) \ln \left(\frac{2 M r-r^{2}-a^{2}}{(M-a)(M+a)}\right) \\
& \left.+C_{1}\right)^{1 / 2}, \pm \sqrt{\frac{\pi^{2}}{4} r^{2}+\pi^{2} 8 a^{2}+C_{2}} \\
& \pm \sqrt{\left(\left(r^{2}+a^{2}\right)+\frac{2 M a^{2}}{r}\right) \phi^{2}+C_{3}} \text {, } \\
& \left. \pm \sqrt{\left(1-\frac{2 M}{r}\right) t^{2}+C_{4}}\right\} \text {, } \\
& \phi(M, 1)=\left\{\frac{1}{2} r^{2}+M^{2}-M r+M \ln (r-M) r\right. \\
& -M^{2} \ln (r-M), \pm \sqrt{\frac{\pi^{2}}{4} r^{2}+C_{2}}, \\
& \left. \pm \sqrt{r^{2} \phi^{2}+C_{3}}, \pm \sqrt{\left(1-\frac{2 M}{r}\right) t^{2}+C_{4}}\right\} \text {. }
\end{aligned}
$$


The deformation retract of the Kerr space into a geodesic $S_{2} \subset K$ is defined by

$$
\begin{aligned}
& \phi(M, c) \\
& =(1-c) \frac{\pi c}{2}\left\{\left(r^{2}-4 M r+\left(2 M r-2 M^{2}\right)\right.\right. \\
& \times \ln \left(-r^{2}-a^{2}+2 M r\right) \\
& +\frac{\tan ^{-1}\left((M-r) / \sqrt{a^{2}-M^{2}}\right)}{\sqrt{a^{2}-M^{2}}} \\
& \times\left(8 M^{3}-6 a^{2} M+2 a^{2} r-4 M^{2} r\right) \\
& +\left(a^{2}-2 M^{2}\right) \ln \left(\frac{2 M r-r^{2}-a^{2}}{(M-a)(M+a)}\right) \\
& \left.+C_{1}\right)^{1 / 2}, \pm \sqrt{\frac{\pi^{2}}{4} r^{2}+\pi^{2} 8 a^{2}+C_{2}} \\
& \pm \sqrt{\left(\left(r^{2}+a^{2}\right)+\frac{2 M a^{2}}{r}\right) \phi^{2}+C_{3}} \\
& \left. \pm \sqrt{\left(1-\frac{2 M}{r}\right) t^{2}+C_{4}}\right\} \\
& +\sin \frac{\pi c}{2}\left\{\frac{1}{2} r^{2}+M^{2}-M r+M \ln (r-M) r\right. \\
& -M^{2} \ln (r-M), \pm \sqrt{\frac{\pi^{2}}{4} r^{2}+C_{2}}, \\
& \left. \pm \sqrt{r^{2} \phi^{2}+C_{3}}, \pm \sqrt{C_{4}}\right\} \text {. }
\end{aligned}
$$

The deformation retract of the Kerr space into a geodesic $S_{3} \subset K$ is defined by

$$
\begin{aligned}
& \phi(M, c) \\
& =\cos \frac{\pi c}{2}\left\{\left(r^{2}-4 M r+\left(2 M r-2 M^{2}\right)\right.\right. \\
& \quad \times \ln \left(-r^{2}-a^{2}+2 M r\right) \\
& \\
& +\frac{\tan ^{-1}\left((M-r) / \sqrt{a^{2}-M^{2}}\right)}{\sqrt{a^{2}-M^{2}}} \\
& \quad \times\left(8 M^{3}-6 a^{2} M+2 a^{2} r-4 M^{2} r\right) \\
& +\left(a^{2}-2 M^{2}\right) \ln \left(\frac{2 M r-r^{2}-a^{2}}{(M-a)(M+a)}\right)
\end{aligned}
$$

$$
\begin{aligned}
& \left.+C_{1}\right)^{1 / 2}, \pm \sqrt{\frac{\pi^{2}}{4} r^{2}+\pi^{2} 8 a^{2}+C_{2}}, \\
& \pm \sqrt{\left(\left(r^{2}+a^{2}\right)+\frac{2 M a^{2}}{r}\right) \phi^{2}+C_{3}}, \\
& \left. \pm \sqrt{\left(1-\frac{2 M}{r}\right) t^{2}+C_{4}}\right\} \\
& +c\left\{\frac{1}{2} r^{2}+M^{2}-M r+M \ln (r-M) r\right. \\
& -M^{2} \ln (r-M), \pm \sqrt{\frac{\pi^{2}}{4} r^{2}+C_{2}}, \\
& \left. \pm \sqrt{C_{3}}, \pm \sqrt{C_{4}}\right\}
\end{aligned}
$$
$K$ :

Now we are going to discuss the folding of the Kerr space

$$
f: K \longrightarrow K
$$

where

$$
f\left(x_{1}, x_{2}, x_{3}, x_{4}\right)=\left(\left|x_{1}\right|, x_{2}, x_{3}, x_{4}\right) .
$$

An isometric folding of the Kerr space into itself may be defined by

$$
\begin{gathered}
\phi(M, c) \\
=\left\{\left(r^{2}-4 M r+\left(2 M r-2 M^{2}\right) \ln \left(-r^{2}-a^{2}+2 M r\right)\right.\right. \\
\quad+\frac{\tan ^{-1}\left((M-r) / \sqrt{a^{2}-M^{2}}\right)}{\sqrt{a^{2}-M^{2}}} \\
\quad \times\left(8 M^{3}-6 a^{2} M+2 a^{2} r-4 M^{2} r\right) \\
\left.+\left(a^{2}-2 M^{2}\right) \ln \left(\frac{2 M r-r^{2}-a^{2}}{(M-a)(M+a)}\right)+C_{1}\right)^{1 / 2}
\end{gathered}
$$




$$
\begin{aligned}
& \longrightarrow\left\{\mid\left(r^{2}-4 M r+\left(2 M r-2 M^{2}\right)\right.\right. \\
& \times \ln \left(-r^{2}-a^{2}+2 M r\right) \\
& +\frac{\tan ^{-1}\left((M-r) / \sqrt{a^{2}-M^{2}}\right)}{\sqrt{a^{2}-M^{2}}} \\
& \times\left(8 M^{3}-6 a^{2} M+2 a^{2} r-4 M^{2} r\right) \\
& +\left(a^{2}-2 M^{2}\right) \\
& \left.\times \ln \left(\frac{2 M r-r^{2}-a^{2}}{(M-a)(M+a)}\right)+C_{1}\right)^{1 / 2} \\
& \pm \sqrt{\frac{\pi^{2}}{4} r^{2}+\pi^{2} 8 a^{2}+C_{2}} \\
& \pm \sqrt{\left(\left(r^{2}+a^{2}\right)+\frac{2 M a^{2}}{r}\right) \phi^{2}+C_{3}} \\
& \left. \pm \sqrt{\left(1-\frac{2 M}{r}\right) t^{2}+C_{4}}\right\} \text {. }
\end{aligned}
$$

$$
\begin{aligned}
& \times \ln \left(-r^{2}-a^{2}+2 M r\right) \\
& +\frac{\tan ^{-1}\left((M-r) / \sqrt{a^{2}-M^{2}}\right)}{\sqrt{a^{2}-M^{2}}} \\
& \times\left(8 M^{3}-6 a^{2} M+2 a^{2} r-4 M^{2} r\right) \\
& +\left(a^{2}-2 M^{2}\right) \\
& \left.\times \ln \left(\frac{2 M r-r^{2}-a^{2}}{(M-a)(M+a)}\right)+C_{1}\right)^{1 / 2} \\
& \pm \sqrt{\frac{\pi^{2}}{4} r^{2}+\pi^{2} 8 a^{2}+C_{2}} \\
& \pm \sqrt{\left(\left(r^{2}+a^{2}\right)+\frac{2 M a^{2}}{r}\right) \phi^{2}+C_{3}} \text {, } \\
& \left. \pm \sqrt{\left(1-\frac{2 M}{r}\right) t^{2}+C_{4}}\right\} \text {, }
\end{aligned}
$$

with

The deformation retract of the folded Kerr space $K$ into the folded $S_{1}$ is

$$
\begin{aligned}
& \phi f:\left\{\mid\left(r^{2}-4 M r+\left(2 M r-2 M^{2}\right)\right.\right. \\
& \times \ln \left(-r^{2}-a^{2}+2 M r\right) \\
& +\frac{\tan ^{-1}\left((M-r) / \sqrt{a^{2}-M^{2}}\right)}{\sqrt{a^{2}-M^{2}}} \\
& \times\left(8 M^{3}-6 a^{2} M+2 a^{2} r-4 M^{2} r\right) \\
& \left.+\left(a^{2}-2 M^{2}\right) \ln \left(\frac{2 M r-r^{2}-a^{2}}{(M-a)(M+a)}\right)+C_{1}\right)^{1 / 2} \\
& \pm \sqrt{\frac{\pi^{2}}{4} r^{2}+\pi^{2} 8 a^{2}+C_{2}} \\
& \pm \sqrt{\left(\left(r^{2}+a^{2}\right)+\frac{2 M a^{2}}{r}\right) \phi^{2}+C_{3}} \text {, } \\
& \left. \pm \sqrt{\left(1-\frac{2 M}{r}\right) t^{2}+C_{4}}\right\} \times I \\
& \longrightarrow\left\{\mid\left(r^{2}-4 M r+\left(2 M r-2 M^{2}\right)\right.\right.
\end{aligned}
$$

$\phi f(M, c)$

$$
\begin{aligned}
=\cos \frac{\pi c}{2}\{\mid & \left(r^{2}-4 M r+\left(2 M r-2 M^{2}\right)\right. \\
& \times \ln \left(-r^{2}-a^{2}+2 M r\right) \\
& +\frac{\tan ^{-1}\left((M-r) / \sqrt{a^{2}-M^{2}}\right)}{\sqrt{a^{2}-M^{2}}} \\
& \times\left(8 M^{3}-6 a^{2} M+2 a^{2} r-4 M^{2} r\right) \\
& +\left(a^{2}-2 M^{2}\right) \\
+\sin \frac{\pi c}{2}\{ & \mid \frac{1}{2} r^{2}+M^{2}-M r+M \ln (r-M) r \\
& \times \sqrt{\left.\ln \left(\frac{2 M r-r^{2}-a^{2}}{(M-a)(M+a)}\right)+C_{1}\right)} \mid, \\
\pm & \sqrt{\frac{\pi^{2}}{4} r^{2}+\pi^{2} 8 a^{2}+C_{2},} \\
\pm & \sqrt{\left(\frac{\left.\left(r^{2}+a^{2}\right)+\frac{2 M a^{2}}{r}\right)}{r}\right) \phi^{2}+C_{3},}
\end{aligned}
$$




$$
\begin{aligned}
& -M^{2} \ln (r-M) \mid, \pm \sqrt{\frac{\pi^{2}}{4} r^{2}+C_{2}}, \\
& \left. \pm \sqrt{r^{2} \phi^{2}+C_{3}}, \pm \sqrt{\left(1-\frac{2 M}{r}\right) t^{2}+C_{4}}\right\} .
\end{aligned}
$$

The deformation retract of the folded Kerr space $K$ into the folded $S_{2}$ is

$\phi f(M, c)$

$$
\begin{aligned}
& =(1-c)\left\{\mid\left(r^{2}-4 M r+\left(2 M r-2 M^{2}\right)\right.\right. \\
& \times \ln \left(-r^{2}-a^{2}+2 M r\right) \\
& +\frac{\tan ^{-1}\left((M-r) / \sqrt{a^{2}-M^{2}}\right)}{\sqrt{a^{2}-M^{2}}} \\
& \times\left(8 M^{3}-6 a^{2} M+2 a^{2} r-4 M^{2} r\right) \\
& +\left(a^{2}-2 M^{2}\right) \\
& \left.\times \ln \left(\frac{2 M r-r^{2}-a^{2}}{(M-a)(M+a)}\right)+C_{1}\right)^{1 / 2}, \\
& \pm \sqrt{\frac{\pi^{2}}{4} r^{2}+\pi^{2} 8 a^{2}+C_{2}} \\
& \pm \sqrt{\left(\left(r^{2}+a^{2}\right)+\frac{2 M a^{2}}{r}\right) \phi^{2}+C_{3}} \text {, } \\
& \left. \pm \sqrt{\left(1-\frac{2 M}{r}\right) t^{2}+C_{4}}\right\} \\
& +\sin \frac{\pi c}{2}\left\{\frac{1}{2} r^{2}+M^{2}-M r+M \ln (r-M) r\right. \\
& -M^{2} \ln (r-M), \pm \sqrt{\frac{\pi^{2}}{4} r^{2}+C_{2}}, \\
& \left. \pm \sqrt{r^{2} \phi^{2}+C_{3}}, \pm \sqrt{C_{4}}\right\} \text {. }
\end{aligned}
$$

The deformation retract of the folded Kerr space $K$ into the folded $S_{3}$ is

$\phi(M, c)$

$$
=\cos \frac{\pi c}{2}\left\{\mid\left(r^{2}-4 M r+\left(2 M r-2 M^{2}\right)\right.\right.
$$

$$
\begin{aligned}
& \times \ln \left(-r^{2}-a^{2}+2 M r\right) \\
& +\frac{\tan ^{-1}\left((M-r) / \sqrt{a^{2}-M^{2}}\right)}{\sqrt{a^{2}-M^{2}}} \\
& \times\left(8 M^{3}-6 a^{2} M+2 a^{2} r-4 M^{2} r\right) \\
& +\left(a^{2}-2 M^{2}\right) \\
& \left.\times \ln \left(\frac{2 M r-r^{2}-a^{2}}{(M-a)(M+a)}\right)+C_{1}\right)^{1 / 2} \\
& \pm \sqrt{\frac{\pi^{2}}{4} r^{2}+\pi^{2} 8 a^{2}+C_{2}} \\
& \pm \sqrt{\left(\left(r^{2}+a^{2}\right)+\frac{2 M a^{2}}{r}\right) \phi^{2}+C_{3}} \text {, } \\
& \left. \pm \sqrt{\left(1-\frac{2 M}{r}\right) t^{2}+C_{4}}\right\} \\
& +c\left\{\frac{1}{2} r^{2}+M^{2}-M r+M \ln (r-M) r\right. \\
& -M^{2} \ln (r-M), \pm \sqrt{\frac{\pi^{2}}{4} r^{2}+C_{2}}, \\
& \left. \pm \sqrt{C_{3}}, \pm \sqrt{C_{4}}\right\}
\end{aligned}
$$

Therefore, the following theorem has been proved.

Theorem 2. The deformation retract of the isometric folding of Kerr space and any folding homeomorphic to this type of folding is different from the deformation retract of Kerr space.

\section{Conclusion}

The deformation retract of the Kerr space has been investigated by making use of Lagrangian equations. The equatorial geodesics of the Kerr space have been discussed. The retraction of this space into itself and into geodesics has been presented. The deformation retraction of the Eguchi-Hanson space is a geodesic which is found to be a great circle. The deformation retract of the isometric folding of Kerr space and any folding homeomorphic to this type of folding is found to be different from the deformation retract of Kerr space.

\section{Conflict of Interests}

The author declares that there is no conflict of interests regarding the publication of this paper. 


\section{Acknowledgment}

The author is deeply indebted to Nasr Ahmed from Mathematics Department at Taibah University for the useful discussions and help during this work.

\section{References}

[1] H. Eschrig, Topology and Geometry for Physics, vol. 822 of Lecture Notes in Physics, Springer, New York, NY, USA, 1st edition, 2011.

[2] E. Bick and F. D. Steffen, Eds., Topology and Geometry in Physics, Lecture Notes in Physics, Springer, 2005.

[3] P. G. Frè, Gravity, a Geometrical Course, Springer, New York, NY, USA, 2012.

[4] D. Rafael, "Consequences of spacetime topology," in Proceedings of the 3rd Canadian Conference on General Relativity and Relativisitc Astrophysics, May 1989.

[5] A. Borde, "Topology change in classical general relativity," http://arxiv.org/abs/gr-qc/9406053.

[6] A. Chamblin, "Some applications of differential topology in general relativity," Journal of Geometry and Physics, vol. 13, no. 4, pp. 357-377, 1994.

[7] M. M. Akbar and G. W. Gibbons, "Ricci-flat metrics with $U(1)$ action and the Dirichlet boundary-value problem in Riemannian quantum gravity and isoperimetric inequalities," Classical and Quantum Gravity, vol. 20, no. 9, pp. 1787-1822, 2003.

[8] J. York, "Black-hole thermodynamics and the Euclidean EINstein action," Physical Review D: Particles and Fields, vol. 33, no. 8, pp. 2092-2099, 1986.

[9] S. W. Hawking, "Gravitational instantons," Physics Letters A, vol. 60, article 81, 1977.

[10] D. N. Page, “Taub-NUT instanton with an horizon," Physics Letters B, vol. 78, no. 2-3, pp. 249-251, 1978.

[11] R. C. Myers and M. J. Perry, "Black holes in higher-dimensional space-times," Annals of Physics, vol. 172, no. 2, pp. 304-347, 1986.

[12] A. Kehagias and K. Sfetsos, "Deviations from the $1 / r^{2}$ Newton law due to extra dimensions," Physics Letters B, vol. 472, no. 1-2, pp. 39-44, 2000.

[13] R. G. Vishwakarma, "Mysteries of the geometrization of gravitation," Research in Astronomy and Astrophysics, vol. 13, no. 12, pp. 1409-1422, 2013.

[14] K. Borsuk, "Sur les rtractes," Fundamenta Mathematicae, vol. 17, p. 220, 1931.

[15] S. A. Robertson, "Isometric folding of Riemannian manifolds," Proceedings of the Royal Society of Edinburgh, vol. 79, no. 3-4, pp. 275-284, 1978.

[16] A. E. El-Ahmady and H. Rafat, "A calculation of geodesics in chaotic flat space and its folding," Chaos, Solitons and Fractals, vol. 30, no. 4, pp. 836-844, 2006.

[17] M. El-Ghoul, A. E. El-Ahmady, and H. Rafat, "Foldingretraction of chaotic dynamical manifold and the VAK of vacuum fluctuation," Chaos, Solitons \& Fractals, vol. 20, no. 2, pp. 209-217, 2004.

[18] M. El-Ghoul, A. E. El-Ahmady, H. Rafat, and M. Abu-Saleem, "The fundamental group of the connected sum of manifolds and their foldings," Journal of the Chungcheong Mathematical Society, vol. 18, no. 2, pp. 161-173, 2005.

[19] H. Rafat and F. Salama, "Fractal dimension and exponential folding of chaotic manifold," Applied Mathematical Sciences, vol. 6, no. 89-92, pp. 4431-4441, 2012.
[20] H. Rafat, "Tiling of topological spaces and their Cartesian product," International Journal of Pure and Applied Mathematics, vol. 27, no. 3, pp. 517-522, 2006.

[21] H. Rafat, "On tiling for some types of manifolds and their foldings," Applied Mathematical Sciences, vol. 3, no. 26, pp. 12751284, 2009.

[22] P. Di Francesco, "Folding and coloring problems in mathematics and physics," Bulletin of the American Mathematical Society, vol. 37, no. 3, pp. 251-307, 2000.

[23] J. Nešetřil and P. Ossona de Mendez, "Folding," Journal of Combinatorial Theory, Series B, vol. 96, no. 5, pp. 730-739, 2006.

[24] W. S. Massey, Algebric Topology: An Introduction, Harcourt Brace and World, New York, NY, USA, 1967.

[25] H. A. Hamm and N. Mihalache, "Deformation retracts of Stein spaces," Mathematische Annalen, vol. 308, no. 2, pp. 333-345, 1997.

[26] H. Rafat, "A spacetime geodesics of the Schwarzschild space and its deformation retract," International Journal of Mathematical Combinatorics, vol. 3, pp. 23-31, 2009.

[27] N. Ahmed and H. Rafat, "Space-time geodesics of the 5D Schwarzschild field and its deformation retract," http:// 128.84.21.199/abs/1405.1057v1. 


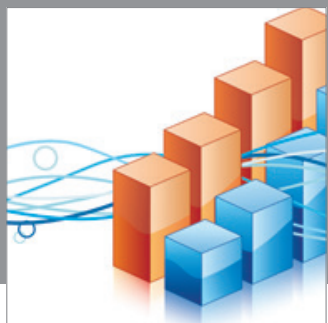

Advances in

Operations Research

mansans

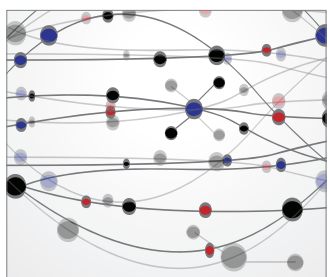

The Scientific World Journal
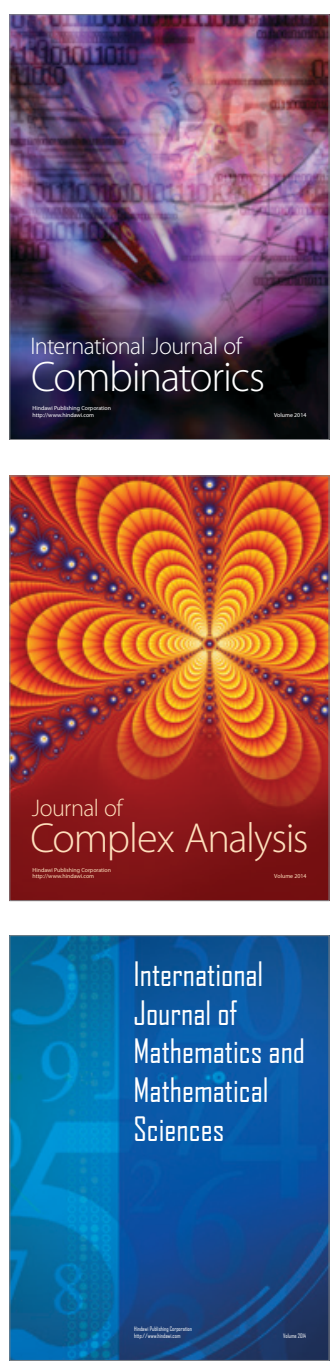
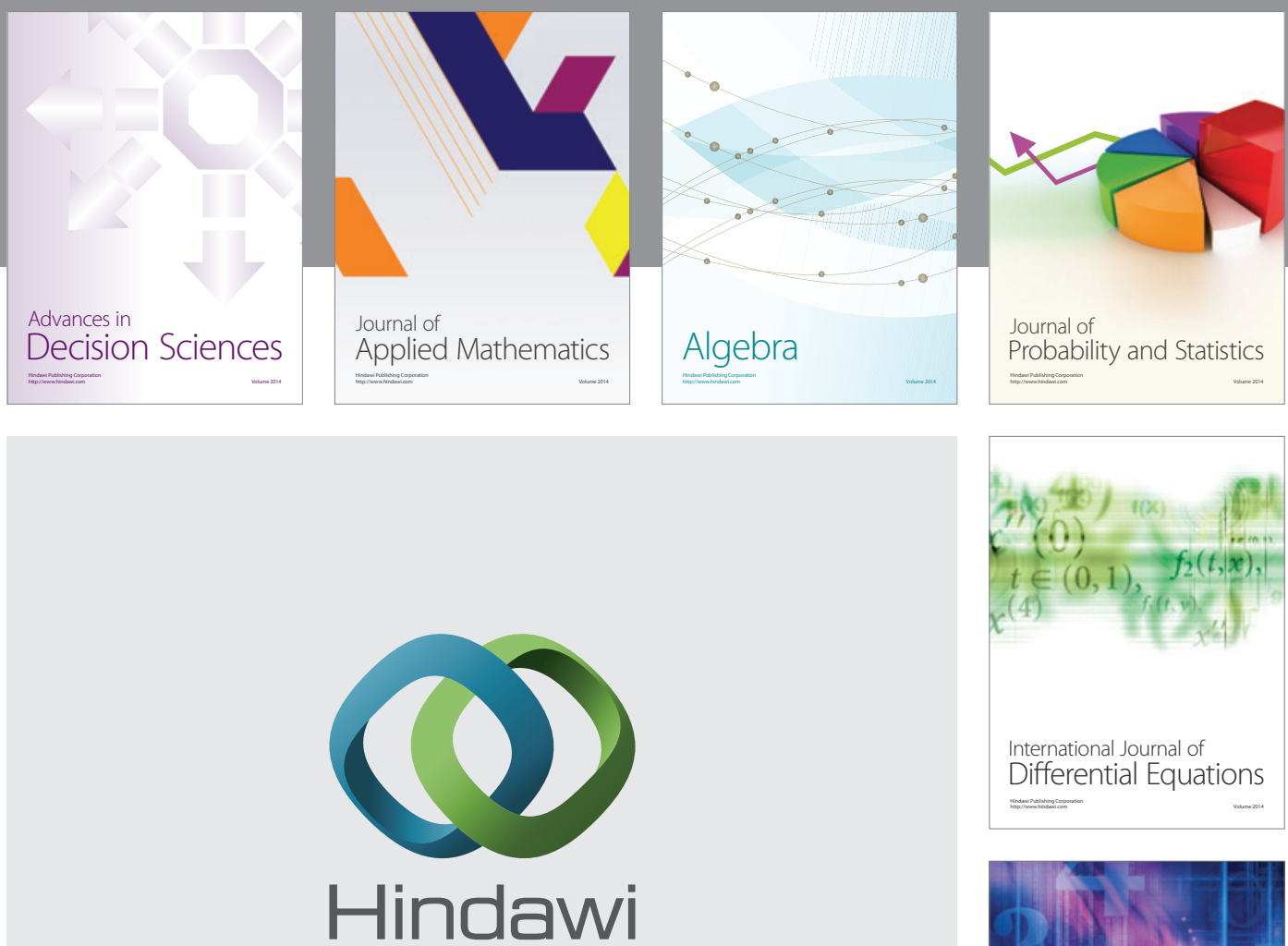

Submit your manuscripts at http://www.hindawi.com
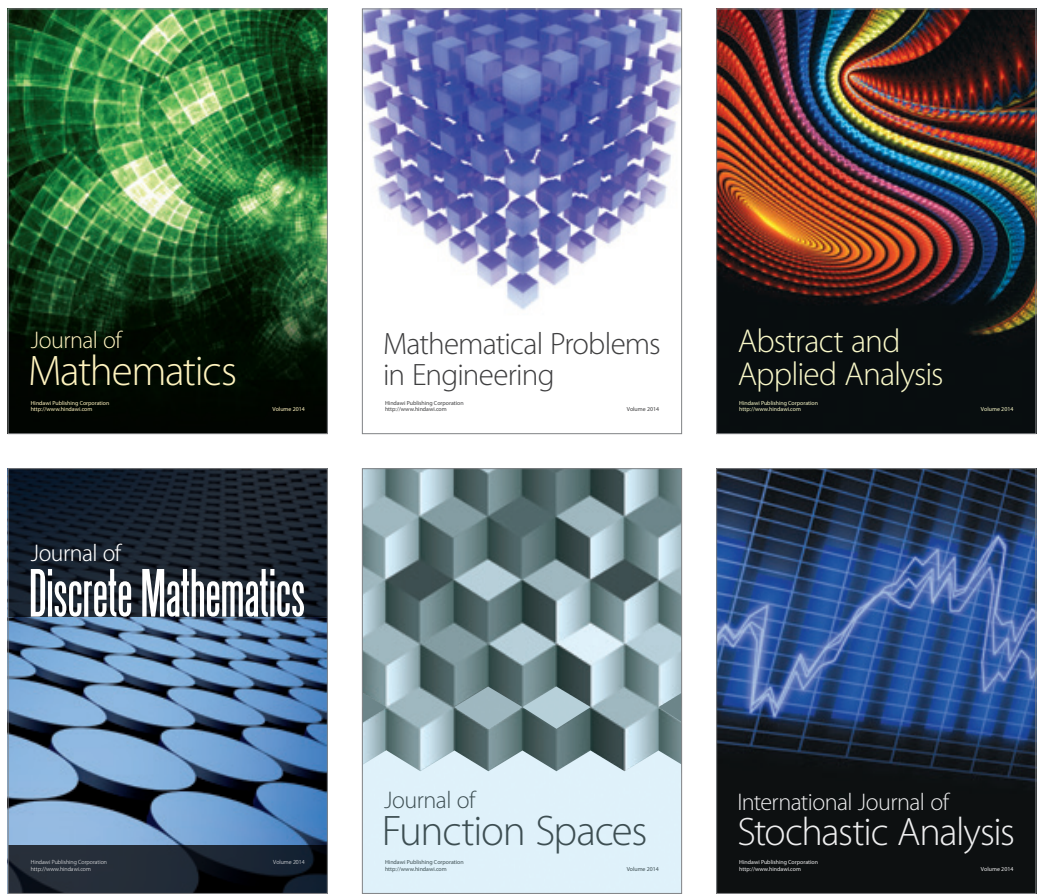

Journal of

Function Spaces

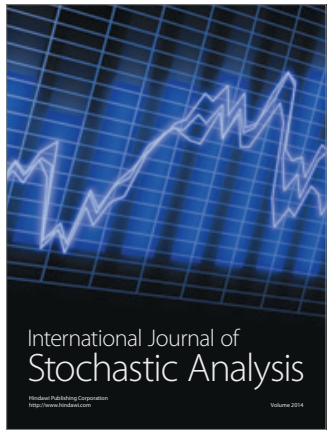

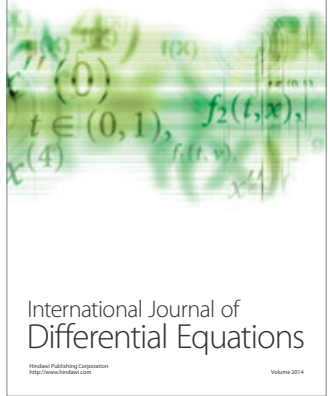
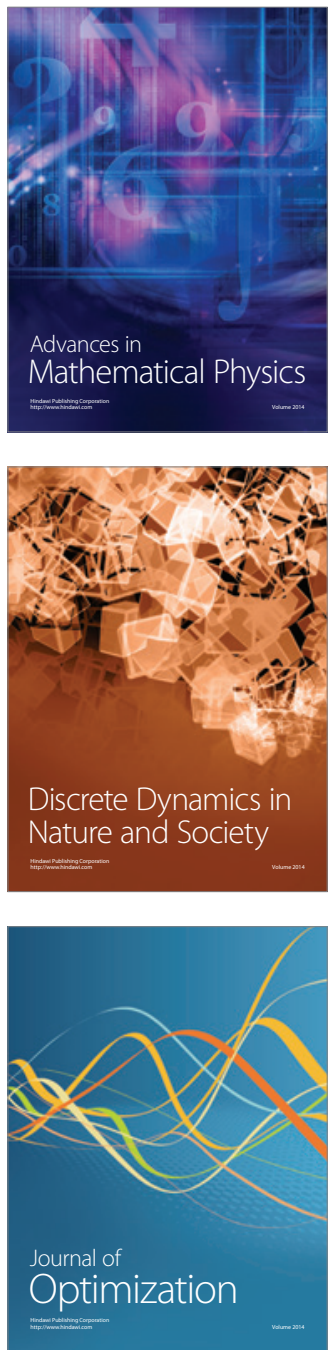